\title{
Over layer sensing using terahertz meta-material split ring resonator
}

\begin{abstract}
Over layer sensing of thin dielectric layers using novel meta-material inspired split rings resonator based sensor is reported in this letter. The sensor is designed to work in terahertz frequency. The contribution of the proposed split ring resonator based terahertz sensor is in the sub-wavelength size simple planar structure design with high quality factor that effectively detects the presence of very thin dielectric sample layers as a function of sensor split gap area coverage.
\end{abstract}

Keyword: Meta-material; Over layer sensing; Split ring resonator 